\title{
A Possible Functional Role of HSP27 as a Molecular Chaperone of Wnt1 in Cell Differentiation of Pleomorphic Adenomas
}

Research Article

Ueda $\mathrm{Y}^{1,2}$, Nakano $\mathrm{K}^{3,4}$, Ochiai $\mathrm{T}^{4}$, Yoshida $\mathrm{W}^{5}$, Sugita $\mathrm{Y}^{5}$, Kubo $\mathrm{K}^{5}$, Maeda $\mathrm{H}^{5}$, Hasegawa $\mathrm{H}^{4}$, Kawakami $\mathrm{T}^{1 *}$

${ }^{1}$ Hard Tissue Pathology Unit, Matsumoto Dental University Institute for Oral Science, Shiojiri, Japan.

${ }^{2}$ Department of Oral Surgery, KKR Hirakata Kohsai Hospital, Hirakata, Japan.

${ }_{3}^{3}$ Department of Oral Pathology and Medicine, Okayama University Graduate School of Medicine, Dentistry and Pharmaceutical Sciences, Okayama, Japan.

${ }^{4}$ Department of Oral Pathology, Matsumoto Dental University School of Dentistry, Shiojiri, Japan.

${ }^{5}$ Department of Oral Pathology, School of Dentistry, Aichi Gakuin University, Nagoya, Japan.

\section{Abstract}

The study focused on the differentiation of parenchymal tumor cells in pleomorphic adenomas. According to our previous published data, Wnt1 is involved in squamous differentiation of basaloid cells in solid nests and small cuboidal cells in duct-like structures during cell differentiation in pleomorphic adenoma. Furthermore, HSP27 sometimes act as a molecular chaperone in neoplastic cells. Thus, immunohistochemistry was performed using Wnt1 and HSP27. A total of 30 cases of pleomorphic adenoma were re-evaluated histologically and categorized based on WHO classification. The mean age of the patients is 51.5 years consisting of 13 males and 17 females. Fourteen tumors were located in the palate, 5 in the parotid gland, 4 in the mandibular gland, 3 in the upper lip, 3 in the buccal mucosa and 1 in other region. Wnt1 and HSP27 expressions were observed under a light microscope. Wnt1 was detected in almost all tumor cells. The basaloid cells with squamous metaplasia and small cuboidal cells forming duct-like structures were strongly positive to Wnt1. The immunofluorescent staining pattern of Wnt1 and HSP27 was consistent with previous results. The results suggest that Wnt1 and HSP27 are involved in tumor cell differentiation of basaloid cells in solid nests and small cuboidal cells in duct-like structures. Moreover, HSP27 is highly involved in cell differentiation such as the formation of squamous metaplasia in solid tumor nests. Since HSP27 expression was similar to Wnt1, it can be inferred that HSP27 works as possible molecular chaperone in Wnt1 signaling.

\section{Introduction}

Pleomorphic adenoma (PA) is the most frequently occurring benign epithelial tumor of the salivary gland [1]. It has a wide spectrum of histopathological features even within the same tumor depending on the location $[2,3]$.

Wnt1 is known to be involved in cell differentiation and proliferation [4]. We showed that Wnt1 is involved in cell differentiation in 2 ways: proliferation of duct-like structures via the b-catenin pathway and differentiation of basaloid cells with squamous metaplasia via the non b-catenin pathway. Furthermore, we also mentioned that Wnt1 is greatly involved in the differentiation of various tissues in PA [5].

HSP27 is a protein that belongs to the small family of heat shock proteins. Although it is generally present in the cytoplasm, the expression shifts to the nucleus in response to stress [6]. It works as molecular chaperone to other proteins during repair.

Hence, we hypothesized the possibility of HSP27 functioning as a molecular chaperone during cell differentiation. Immunohistochemistry was performed in sections of PA and the results were

*Corresponding Author:

Toshiyuki Kawakami,

Professor, Hard Tissue Pathology Unit, Department of Hard Tissue Research, Matsumoto Dental University Graduate School of Oral Medicine, Shiojiri, 399-0781, Japan. Tel: $+81-263-51-2035$

E-mail:kawakami@po.mdu.ac.jp.

Received: September 26, 2016

Accepted: October 18, 2016

Published: October 19, 2016

Citation: Ueda Y, Nakano K, Ochiai T, Yoshida W, Kawakami T, et al., (2016) A Possible Functional Role of HSP27 as a Molecular Chaperone of Wnt1 in Cell Differentiation of Pleomorphic Adenomas. Int J Dentistry Oral Sci. 3(10), 340-343. doi: http://dx.doi.org/10.19070/2377-8075-1600069

Copyright: Kawakami $T^{\circ}$ 2016. This is an open-access article distributed under the terms of the Creative Commons Attribution License, which permits unrestricted use, distribution and reproduction in any medium, provided the original author and source are credited. 
observed under the light microscope.

\section{Materials and Methods}

Samples were obtained from the Department of Oral Pathology, School of Dentistry, Aichi Gakuin University. Histopathological re-evaluation of the files diagnosed as PA was done and 30 cases of PA with typical morphology based on WHO classification were utilized (Table 1). Briefly, the specimens were fixed in $4 \%$ neutral buffered formalin solution, embedded in paraffin blocks, serially sectioned into 4 micron thickness, dehydrated in series of alcohol, stained with $\mathrm{HE}$ and re-examined under the light microscope.

Fluorescence immunohistochemistry (FIHC) using rabbit polyclonal Wnt1 antibody (anti-Wnt1, Abcam, Cambridge, UK; $1 / 100$ ) and mouse monoclonal HSP27 antibody (anti-HSP27, Abcam, Cambridge, UK; 1/100) were carried out. After deparaffinization, specimens were pre-treated in citrate buffer (citric acid buffer, pH 6.0, Mitsubishi Chemical Medience, Tokyo, Japan) placed in microwave for $1 \mathrm{~min}$. Serum-free protein (Dako, Japan Co., Ltd, Tokyo, Japan) was used for blocking at room temperature for $30 \mathrm{~min}$. The primary antibodies (rabbit polyclonal Wnt1 and mouse monoclonal HSP27 antibodies) were each diluted with CanGetSignal (Toyobo, Osaka, Japan) in 100-fold and incubated for 16 hours at $4^{\circ} \mathrm{C}$. For secondary antibodies, donkey anti-rabbit IgG H\&L (Alexa Fluor 594; Abcam, Cambridge, UK; 1/200) and donkey anti-mouse IgG H\&L (Alexa Fluor 488, Abcam, Cambridge, UK, 1/200) were each diluted in CanGetSignal (Toyobo, Osaka, Japan) in 200-fold and allowed to react at room temperature for $1 \mathrm{hr}$. Nuclear stain was revealed using $1 \mathrm{mg} / \mathrm{ml}$ DAPI incubated for $3 \mathrm{~min}$. Then after, specimens were washed with TBS and mounted using Fluorescent Mounting Medium (Dako Japan Co., Ltd., Tokyo, Japan).
The study was approved by the Ethics Committee of AichiGakuin University, School of Dentistry under the title 'Basic, Clinical and Pathological Research in the Elucidation of Pathogenesis and Development of Diagnostic Method of Salivary Gland Tumors' (Number 284).

\section{Results}

Although Wnt1 was expressed by many tumor cells, strong positive expression was primarily seen in solid tumor nests. Moreover, increased expression was observed in areas were cells differentiated into squamous epithelial-like cells (Figure 1-a,b). Wnt1 was localized in small cuboidal cells in the outer lumen forming ductlike structures. The expression seemed to decrease in polygonal cells (Figure 2-a). Wnt1 was strongly expressed by basaloid cells showing squamous metaplasia however the expression decreased in squamous-like cells (Figure 3-a).

HSP27 was expressed by many tumor cells and the positive reaction was observed strongly in solid tumor nests (Figure 1-c, d). Tumor cells that form duct-like structures expressed intense HSP27 in the cytoplasm of tumor cells outside the lumen. Positive nuclear reactions were also noted (Figure 2-b). Positive reaction was observed in the cytoplasm of many squamous-like tumor cells inside the solid nests. Those tumor cells also showed partial positive reaction in the nucleus. In particular, intense expression was observed in basaloid cells during their transition into keratinocytes (Figure 3-b).

The expressions of Wnt1 and HSP27 tend to be similar and this was evident in the superimposition of the localization of Wnt1 and HSP27 as shown by double immunofluorescence staining (Figures 2-c, d, 3-c, d).

Table 1. Cases Examined.

\begin{tabular}{|l|l|ll|}
\hline Age & Sex & Location & \\
\hline Average 51.5 & Male 13 & Palate & 14 \\
& Female 17 & Parotid & 5 \\
& & Mandibular gland 4 \\
& & Upper lip & 3 \\
& & Buccal mucosa & 3 \\
& & Other & 1 \\
\hline
\end{tabular}

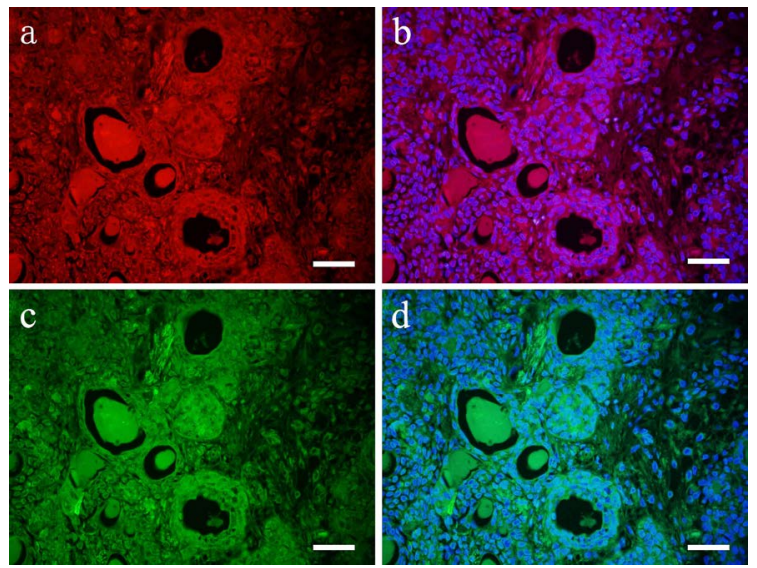

Figure 1. FIHC expressions of Wnt1 (a, b) and HSP27 (c, d) in tumor tissues. a: Wnt1; b: Wnt1 + DAPI; c: HSP27; d: HSP27 + DAPI (scale bar $=50 \mu \mathrm{m})$. 

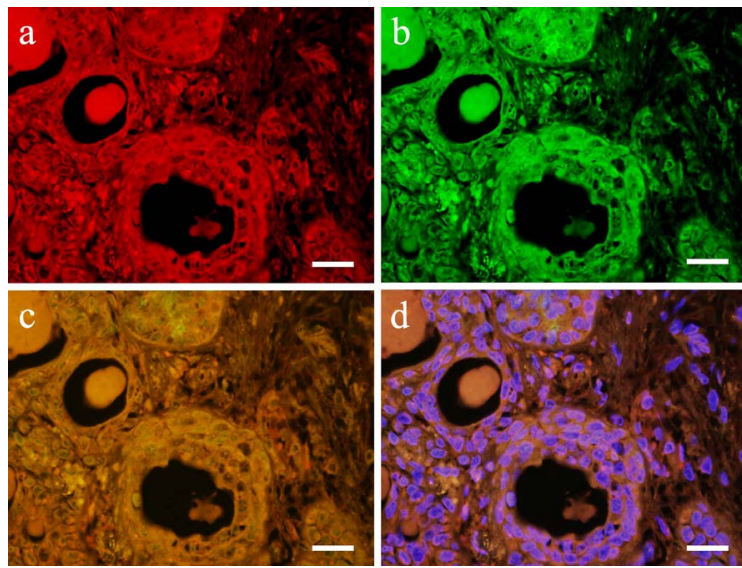

Figure 2. FIHC localization in duct-like structures. a: Wnt1; b: HSP27 + DAPI; c: Wnt1 + HSP27; d: Wnt1 + HSP27 + DAPI (scale bar $=25 \mu \mathrm{m})$.
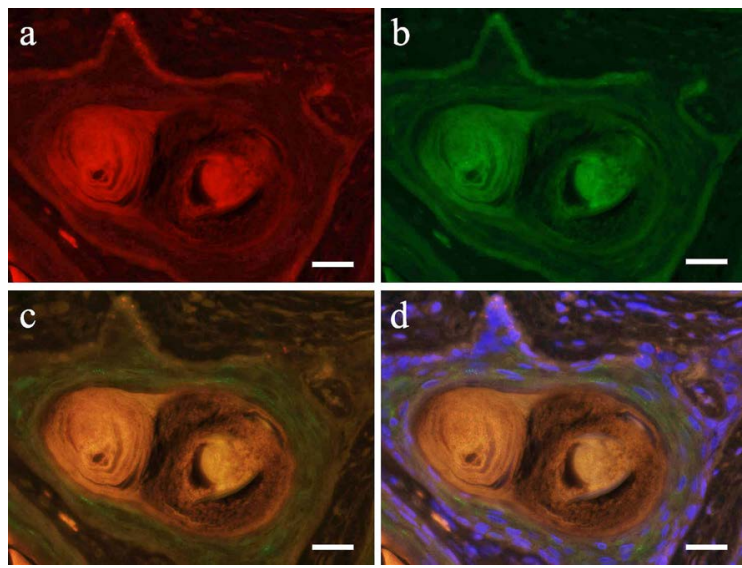

Figure 3. FIHC localization in squamous metaplasia area. a: Wnt1; b: HSP27 + DAPI; c: Wnt1 + HSP27; d: Wnt1 + HSP27 + DAPI (scale bar $=25 \mu \mathrm{m})$.

\section{Discussion}

HSP is a major protein expressed in various organs and tissues in response to cytotoxic stimuli and mechanical stress. HSPs are not only related to heat shock but are also stimulated by other various pathological conditions like radiation, enzyme, heavy metals, arsenic, ethanol and stress caused by active enzymes and amino acid derivatives [7]. It is also involved in cell injury, defense, repair and in homeostasis [8]. HSPs are classified according to molecular weight, size and structural type. In particular, HSP27 has been involved in various cellular differentiation [9]. Many of the HSPs are expressed in response to cellular stress. HSPs have been associated with suppression of protein breakdown and are know to function in the repair of degraded protein. The low molecular weight HSPs have been regarded as molecular chaperones associated in cell growth and differentiation [10-13]. Although Fujita et al. mentioned that HSP27 is highly involved in cell differentiation in ameloblastoma [14], our search did not reveal a study of HSP in tumors affecting the oral cavity.

Wnt1 has been investigated in various areas, especially in oral tumors such as calcified cystic odontogenic tumor, odontoma, calcifying epithelioma, ameloblastoma, craniopharyngioma, etc. Wnt signaling pathway has been associated with ghost and shadow cells. Ghost cells showed strong positive reaction to antibodies against hair proteins localized in the nucleus and cytoplasm through b-catenin pathway. Epithelial cells also showed increased expression and showed localization of Lef-1 in the nucleus. Involvement of Wnt signaling pathway is believed to be responsible for the appearance of these cells [12]. As described above, Wnt is a typical signal that controls the differentiation of cell and tissue growth. In addition, to homeostasis and cell proliferation, researches are on its way to determine its association in suppressing tissue differentiation in tumors [13].

Our results showed that Wnt1 is involved in cell differentiation in PA. In particular, Wnt1 was detected in peripheral cells in squamous metaplasia and in small cuboidal cells in duct-like structures. It was also believed that Wnt signaling is mediated by b-catenin pathway. Although a strong reaction was observed in basaloid cells surrounding squamous metaplasia, a weak reaction during the transition to prickle cell layer was also reported [5].

Wnt1 expression observed in this study is consistent with our previous report. Furthermore, HSP27 was also localized in the same sites with Wnt1. Fujita et al. [14] previously mentioned that HSP27 is expressed during the differentiation of cells into squamous metaplasia and said to be deeply involved in cell differentiation in ameloblastoma. Accordingly, the same expression in squamous metaplasia was also observed in PA. Since this is consistent with Wnt1 expression, HSP27 is presumed to have worked as a molecular chaperone of Wnt1. Furthermore, since Wnt1 and HSP27 were both expressed by cells forming duct-like structures and those that underwent squamous metaplasia, it is 
possible that HSP27 worked as a molecular chaperone of Wnt1. This research material is only limited 30 cases. However, the most staining results of almost cases indicated the same tendency. More examinations using large number of cases may be necessary, but I believe that the present result is effective as a temporary quarrel report. In near future date, we will present the large number cases examination results of the relation research.

\section{Conclusions}

Pleomorphic adenoma (PA) has a wide spectrum of histopathological features even within the same tumor depending on the location. Regarding the cell differentiation, Wnt1 is known to be involved in PA. Further, HSP27 works as molecular chaperone to other proteins during repair. Thus, we hypothesized the possibility of HSP27 functioning as a molecular chaperone during cell differentiation. Our present results suggested that Wnt1 and HSP27 are involved in cell differentiation in squamous metaplasia and duct-like structures in PA, Number of cases of this subject of research was 30 and a limited number, but the above mentioned result believes indicated directionality not to be wrong. Thus, HSP27 may have function as a molecular chaperone of Wnt1 in PA.

\section{Acknowledgement}

This research was supported by the Japan Society for the Promotion of Scientific Research, Basic Research C (\# 23592951, \#26463031).

\section{References}

[1]. Eveson JW, Kusafuka K, Stenman G, Nagano T (2005) Pleomorphic adenoma. In: World Health Organization Classification of Tumours. Pathology and Genetics of the Head and Neck Tumours. IARC Press, Lyon, France. 254-260.
[2]. Akiyama W (2011) A histopathological and immunohistochemical study of cartilage-like tissue formation in pleomorphic adenoma: Comparative study of the major and minor salivary gland adenomas. Int J Oral Med Sci. 10(4): 384-399.

[3]. Nakano K, Watanabe T, Shimizu T, Kawakami T (2007) Immunohistochemical characteristics of bone forming cell in pleomorphic adenoma. Int J Med Sci 4(5): 264-266.

[4]. Arend RC, Londoño-Joshi AI, Straughn JM Jr, Buchsbaum DJ (2013) The Wnt/ $\beta$-catenin pathway in ovarian cancer: A review. Gynecol Oncol 131(3): 772-779.

[5]. Okuda Y, Nakano K, Suzuki K, Sugita Y, Kubo K, et al., (2014) Wnt signaling as a possible promoting factor of cell differentiation in pleomorphic adenomas. Int J Med Sci 11(9): 971-978.

[6]. Milton J (1990) Heat shock proteins. J Biol Chem 265(21): 12111-12114.

[7]. Saantoro MG (2000) Heat shock factors and the control of the stress response. Biochem Pharmacol 59(1): 55-63.

[8]. Gething MJ, Sambrook J (1992) Proteins folding in the cell. Nature 355(6355): 33-45.

[9]. de Thonel A, Vandekerckhove J, Lanneau D, Selvakumar S, Courtois G, et al.,(2010) HSP27 controls GATA-1 protein level during erythroid cell diffentiation. Blood 116(1): 85-96.

[10]. Parsell DA, Linndoquist S (1993) The function of heat-shock proteins in stress tolerance: Degradation and reactivation of damaged proteins. An Rev Genet 27: 437-496.

[11]. Hendrick JP, Hartl F (1993) Molecular Chaperone Functions of Heat-Shock Proteins. Ann Rev Biochem 62: 349-384.

[12]. Siar CH, Nagatsuka H, Nakano K, Ham PP, Nagatsuka H, et al., (2012) Differential expression of canonial and non-canonial Wnt ligands in ameloblatoma. J Oral Pathol Med 41(4): 332-339.

[13]. Li PP, Wang X (2013) Role of signaling pathways and miRNAs in chronic lymphocytic leukemia. Chin Med J 126(21): 4175-4182.

[14]. Fujita M, Nakano K, Funato A, Sugita Y, Kubo K, et al., (2013) Heat shock protein 27 expression and cell differentiation in ameloblastomas. Int J Med Sci. 10: 1271-1277. 